\title{
A Vehicle Target Searching Method
}

\author{
Yong Sun, Wenwei Li, Tiezheng Liu and Jirong Xue \\ China Research and Development Academy of Machinery Equipment; Beijing 100089; China \\ sunyong1984@gmail.com
}

Keywords: Target searching, vehicle, observing and sighting instrument, method.

Abstract. Based on some assuming and definition, this paper propose a method of vehicle target searching, this method include calculate the scanning time, the probability of finding the target when the target intersection with the sight center line, and the probability of finding a target within a time interval, this method is easy and practicality.

\section{Introduction}

When observing and sighting instrument search target, there are many factors affect the probability of finding the target at the given time, in addition to people, also: instrument performance, search range, target exposed visual area, target distance, background complexity, weather visibility, whether the target movement, terrain conditions, etc., when simulate the optical aiming instrument search target process, how to consider these factors scientifically and reasonably, how to determine the parameters of the model based on the training data is worthy of serious study.

\section{On the vision of the existing research results}

(1) When the human eye observation, the sight of the central area $\left(5^{\circ} \times 5^{\circ}\right)$ is gaze area, and is the main area of concern, the attention of the people outside the region is greatly reduced.

(2) In the context of other conditions determined, when the human eye gaze, the probability of its discovery or identify the target is non-linear function of time, the function has two important features:

a) The probability of finding the target increases monotonically with time;

b) When $t \geq 1$, the function tends to a constant value, that is, the time is not long that the function tends to a constant value.

(3) Industrial use of JOHNSON judgment

When the human eye gaze target (i.e., the target is located in the central area of the sight line) the probability of finding or identifying the target can be referred to in Table 1.

Table 1 Industrial use of JOHNSON judgment (one-dimensional target)

\begin{tabular}{|c|l|c|}
\hline $\begin{array}{c}\text { Recognitio } \\
\mathrm{n} \text { level }\end{array}$ & \multicolumn{1}{|c|}{ Meaning } & $\begin{array}{c}\text { The minimum size of the } \\
\text { number of circle } \mathrm{N}_{50}\end{array}$ \\
\hline probe & $\begin{array}{l}\text { There is a goal, to distinguish the target from the } \\
\text { background }\end{array}$ & 1 \\
\hline distinguish & $\begin{array}{l}\text { Identify which category the target belongs to } \\
\text { confirm }\end{array}$ & $\begin{array}{l}\text { Recognize the target and determine the type of the } \\
\text { target. }\end{array}$ \\
\hline
\end{tabular}

$\mathrm{N}_{50}-50 \%$ probability of discovery (identification) target required pairs number.

Table 2 target transfer probability function

\begin{tabular}{|c|l|l|l|l|l|l|l|l|}
\hline Detection probability & 1 & 0.95 & 0.8 & 0.5 & 0.3 & 0.1 & 0.02 & 0 \\
\hline Factor (N) & 3 & 2 & 1.5 & 1 & 0.75 & 0.5 & 0.25 & 0 \\
\hline
\end{tabular}

From the above Table 2, the fitting of the detection probability formula

$$
P(N)=1-e^{-\lambda N^{2}}
$$

Where the coefficient factor $\lambda=\ln 2$.

The detection probability calculated by the above fitting formula is shown in Table 3. From the data in the table, it can be known that the fitting formula is realistic. 
Table 3 detection probability

\begin{tabular}{|l|l|l|l|l|l|l|l|l|}
\hline Detection probability & 1 & 0.95 & 0.8 & 0.5 & 0.3 & 0.1 & 0.02 & 0 \\
\hline Fitting formula & 0.998 & 0.9375 & 0.7898 & 0.5 & 0.323 & 0.159 & 0.04 & 0 \\
\hline Factor $(\mathrm{N})$ & 3 & 2 & 1.5 & 1 & 0.75 & 0.5 & 0.25 & 0 \\
\hline
\end{tabular}

Considering the difference of detection probability under different background, as shown in Table 4. Table 4 the number of circle $\left(\mathrm{N}_{50}\right)_{D}$ and pixel points $\left(\mathrm{N}_{50}\right)_{\mathrm{L}}$ approximation of the target acquisition in different background

\begin{tabular}{|c|c|c|c|}
\hline $\begin{array}{c}\text { Background } \\
\text { clutter }\end{array}$ & Examples & $\left(\mathrm{N}_{50}\right)_{\mathrm{D}}$ & $\left(\mathrm{N}_{50}\right)_{\mathrm{L}}$ \\
\hline No clutter & $\begin{array}{c}\text { Bright source, movement, highly } \\
\text { visible target }\end{array}$ & $<<0.5$ & 1 \\
\hline Low clutter & Goals in the field, on the road & 0.5 & 1 \\
\hline $\begin{array}{c}\text { Moderate } \\
\text { clutter }\end{array}$ & $\begin{array}{c}\text { The tank is in the desert of the tank } \\
\text { like bush. }\end{array}$ & 1.0 & 2 \\
\hline $\begin{array}{c}\text { High clutter } \\
\text { Vehicle in an array of similar } \\
\text { vehicles. }\end{array}$ & 2.0 & 4 \\
\hline
\end{tabular}

\section{Assumptions}

3.1 Viewer search target used time is related to the needed search area, the greater the area, the longer it takes to traverse a search area

3.2 When viewer search target in the search area, he has the self-adaptive ability on sighting the angular velocity of the observing and sighting instrument, that is simple background area, searching angular velocity will increase, while the background is complex area search speed will slow down, which is assumed in process of viewer scanning and searching, the target stay long enough in the sight of the central area.

\section{The scanning time}

Given the search area $\beta_{0} \times \varepsilon_{0}=S_{0}$, observing and sighting instrument view angle $\psi_{0} \times \theta_{0}$, and the complexity of the search area average background, calculate the time $\mathrm{T}$ of once scan the search area, as shown in Fig. 1.

Let observing and sighting instrument scanning angular velocity in azimuth and elevation direction is equal to the average of $\mathrm{w}$, then

$$
T=\left\{\begin{array}{c}
\frac{\beta_{0}-\psi_{0}}{W}\left(\left[\frac{\varepsilon_{0}-\theta_{0}}{\theta_{0}}\right]+1\right)+\frac{\varepsilon_{0}-\theta_{0}}{W} \text { when } \frac{\varepsilon_{0}-\theta_{0}}{\theta_{0}} \text { not int eger } \\
\frac{\beta_{0}-\psi_{0}}{W} \cdot \frac{\varepsilon_{0}-\theta_{0}}{\theta_{0}}+\frac{\varepsilon_{0}-\theta_{0}}{W} \quad \text { when } \frac{\varepsilon_{0}-\theta_{0}}{\theta_{0}} \text { is int eger }
\end{array}\right.
$$

$\mathrm{w}$ is determined by the training data. (Where the brackets are rounded down)

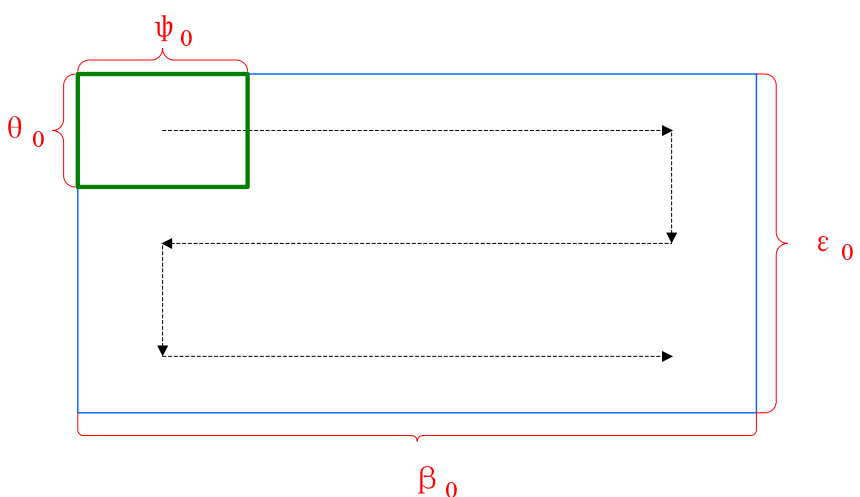

Fig. 1 calculate scanning time 


\section{Calculated the probability of finding the target when the target intersection with the sight center line.}

$$
P=1-e^{-\mu^{\prime} S}
$$

Where $\mu$ ' is the adjustment factor, if the complexity of the background is $i$, the needed target area is $S_{i}$ when $50 \%$ probability detect the target, the adjustment factor is $\mu_{i}^{\prime}=\ln 2 / S_{i} . S^{\prime}$ is the exposed area on the display, which is the number of pixels, as shown in the following

$$
S^{\prime}=\frac{w}{2 d \cdot \operatorname{tg} \theta_{w} / 2} \cdot \frac{l}{2 d \cdot \operatorname{tg} \theta_{l} / 2} \cdot n_{l} \cdot n_{w}
$$

Where $\theta_{\mathrm{w}}$ is transverse field view angle of simulator concept observing and sighting instrument, $\theta_{1}$ is longitudinal field view angle of simulator concept observing and sighting instrument, $d$ is the target distance, $\mathrm{w}$ is the target exposed part longitudinal dimension on the display, 1 is the target exposed part longitudinal dimension on the display, $\mathrm{n}_{\mathrm{w}}$ is the number of display horizontal pixels, $\mathrm{n}_{\mathrm{l}}$ is the number of display longitudinal pixels, as shown in Fig. 2.

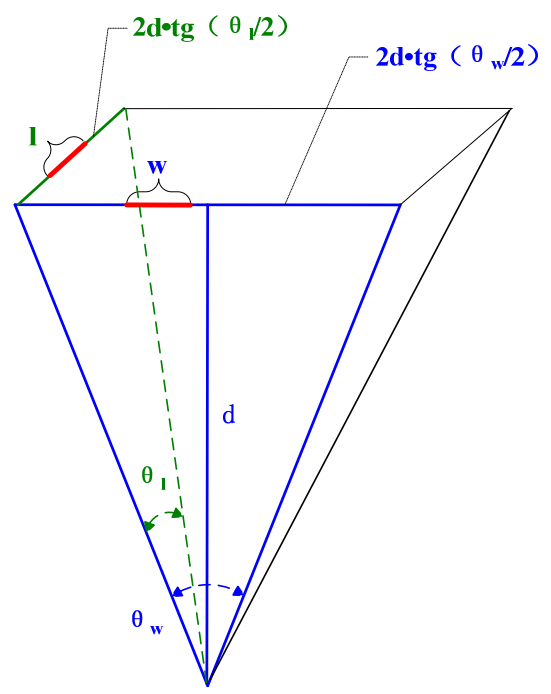

Fig. 2 calculate the probability of finding the target

\section{The probability of finding a target within a time interval $(0, t)$}

Let observing and sighting instrument start the search target at time $\mathrm{t}=0$, calculate the probability of finding the target in the time interval $(0, \mathrm{t})$.

$$
P(\xi \leq t)=\left\{\begin{array}{lrl}
\frac{t}{T}\left(1-e^{-\lambda \theta}\right) & \text { when } t \leq T \\
\left(1-e^{-\lambda \theta}\right)+\frac{t-T}{T}\left(1-e^{-\lambda \theta}\right) e^{-\lambda \theta} & \text { when } & T<t \leq 2 T \\
P(\xi \leq k t)+P(k T<\xi \leq(k+1) T) & \text { when } & k T<t \leq(k+1) T \\
=P(\xi \leq k t)+[1-P(\xi \leq k T)] \frac{t-k T}{T}\left(1-e^{-\lambda \theta}\right) & \\
=\left(1-e^{-k \lambda \theta}\right)+e^{-k \lambda \theta} \frac{t-k T}{T}\left(1-e^{-\lambda \theta}\right) &
\end{array}\right.
$$

\section{The probability of finding a target within a time interval $\left(t_{1}, t_{2}\right)$}

Let observing and sighting instrument start the search target at time $\mathrm{t}=0$, and not find the target before $t \leq t_{1}$, calculate the probability of finding the target in the time interval $\left(t_{1}, t_{2}\right)$. 


$$
P\left(t_{1}<\xi \leq t_{2}\right)=P\left(\xi \leq t_{2}\right)-P\left(\xi \leq t_{1}\right)
$$

\section{References}

[1] Li Wen-wei, Meng Hong, Sun Yong, etc.. Simulation of Optical Imaging Reconnaissance to Ground. 2012 National Conference on Information Technology and Computer Science (CITCS2012), 2012

[2] HAN Zhi-jun, ZHANG Ren-you, LI Da-peng. Study on Combat Process Simulation of Tank Element. FIRE CONTROL AND COMMAND CONTROL: 2007, 32(12)

[3] Xu Da-jie, Song Xian-long, Zhao Duan-bo. The Study of Probability of Detecting Targets in the Tank. FIRE CONTROL \& COMMAND CONTROL: 2003, 28(z1)

[4] Han Zhi-jun, Xu Ke-hu, Li Meng. Simulation Study on the CGF Entities of Tank Element. JOURNAL OF SYSTEM SIMULATION: 2004, 16(7)

[5] HU Xiao-yun. Study on Reconnaissance Functional Simulation Model of Visible Light Reconnaissance Equipment. COMMAND CONTROL \& SIMULATION: 2007, 29(1)

[6] LIANG Dong-ming, ZHANG Man-ju, ZHANG Yan-xiu, etc. Equivalent calculation analysis for operating range of visible light reconnaissance system. Modern Electronics Technique: 2013, $36(1)$

[7] HU Xiao-yun. Study on Reconnaissance Functional Simulation Model of Visible Light Reconnaissance Equipment. COMMAND CONTROL \& SIMULATION: 2007, 29(1) 